\title{
ISOMETRIC DILATIONS AND VON NEUMANN INEQUALITY FOR FINITE RANK COMMUTING CONTRACTIONS
}

\author{
SIBAPRASAD BARIK, B. KRISHNA DAS, AND JAYDEB SARKAR
}

\begin{abstract}
Motivated by Ball, Li, Timotin and Trent's Schur-Agler class version of commutant lifting theorem, we introduce a class, denoted by $\mathcal{P}_{n}(\mathcal{H})$, of $n$-tuples of commuting contractions on a Hilbert space $\mathcal{H}$. We always assume that $n \geq 3$. The importance of this class of $n$-tuples stems from the fact that the von Neumann inequality or the existence of isometric dilation does not hold in general for $n$-tuples, $n \geq 3$, of commuting contractions on Hilbert spaces (even in the level of finite dimensional Hilbert spaces). Under some rank-finiteness assumptions, we prove that tuples in $\mathcal{P}_{n}(\mathcal{H})$ always admit explicit isometric dilations and satisfy a refined von Neumann inequality in terms of algebraic varieties in the closure of the unit polydisc in $\mathbb{C}^{n}$.
\end{abstract}

\section{INTRODUCTION}

In this paper we deal with the problem of isometric dilations and (refined) von-Neumann inequality for $n$-tuples $(n \geq 3)$ of commuting contractions. For notational convenience, we denote by $\mathcal{T}^{n}(\mathcal{H})$ the set of all ordered $n$-tuples of commuting contractions on a Hilbert space $\mathcal{H}$ :

$$
\mathcal{T}^{n}(\mathcal{H})=\left\{\left(T_{1}, \ldots, T_{n}\right): T_{i} \in \mathcal{B}(\mathcal{H}),\left\|T_{i}\right\| \leq 1, T_{i} T_{j}=T_{j} T_{i}, 1 \leq i, j \leq n\right\},
$$

where $\mathcal{B}(\mathcal{H})$ denotes the set of all bounded linear operators on $\mathcal{H}$. Let $V=\left(V_{1}, \ldots, V_{n}\right) \in$ $\mathcal{T}^{n}(\mathcal{K})$ be a tuple of commuting isometries (that is, $V_{i}^{*} V_{i}=I_{\mathcal{K}}$ for all $i=1, \ldots, n$ ) on a Hilbert space $\mathcal{K}$, and let $\mathcal{K} \supseteq \mathcal{H}$ (or, $\mathcal{H}$ is isometrically embedded in $\mathcal{K}$ ). We say that $V$ is an isometric dilation of $T \in \mathcal{T}^{n}(\mathcal{H})$ if

$$
T^{k}=\left.P_{\mathcal{H}} V^{\boldsymbol{k}}\right|_{\mathcal{H}} \quad\left(\boldsymbol{k} \in \mathbb{Z}_{+}^{n}\right),
$$

where $P_{\mathcal{H}}$ denotes the orthogonal projection of $\mathcal{K}$ onto $\mathcal{H}$ and

$$
\mathbb{Z}_{+}^{n}=\left\{\boldsymbol{k}=\left(k_{1}, \ldots, k_{n}\right): k_{i} \in \mathbb{Z}_{+}, i=1, \ldots, n\right\},
$$

and for each multi-index $\boldsymbol{k} \in \mathbb{Z}_{+}^{n}$ and commuting tuple $A=\left(A_{1}, \ldots, A_{n}\right)$ on a Hilbert space $\mathcal{L}$ we denote $A^{k}=A_{1}^{k_{1}} \cdots A_{n}^{k_{n}}$. It is well known that the existence of an isometric dilation of $T \in \mathcal{T}^{n}(\mathcal{H})$ guarantees [21] the von-Neumann inequality for $T$ :

$$
\left\|p\left(T_{1}, \ldots, T_{n}\right)\right\|_{\mathcal{B}(\mathcal{H})} \leq \sup \left\{|p(\boldsymbol{z})|: \boldsymbol{z} \in \overline{\mathbb{D}}^{n}\right\},
$$

for all $p \in \mathbb{C}\left[z_{1}, \ldots, z_{n}\right]$. Now one knows on one hand the existence of isometric dilations of $n$-tuples in $\mathcal{T}^{n}(\mathcal{H}), n=1,2$, is guaranteed by the celebrated dilation theory of Sz.-Nagy and

2010 Mathematics Subject Classification. 47A13, 47A20, 47A56, 47B38, 14M99, 46E20, 30H10.

Key words and phrases. von Neumann inequality, isometric dilations, inner multipliers, Schur-Agler class, Hardy space, distinguished variety. 
Foias and Ando. On the other hand, in general, neither the existence of isometric dilation nor the von Neumann inequality holds for tuples in $\mathcal{T}^{n}(\mathcal{H}), n>2$ (even in the case of $\operatorname{dim} \mathcal{H}<\infty)$. For instance, see the counterexamples by Varopoulous [23], Crabb and Davie [7] and Parrott [22].

An intriguing question therefore is to identify those $n$-tuples in $\mathcal{T}^{n}(\mathcal{H}), n \geq 3$, which admit isometric dilation (and also satisfy the von Neumann inequality over a variety in $\overline{\mathbb{D}}^{n}$ or $\mathbb{D}^{n}$ in the sense of [1], [10], [11] and [5]). This has turned into one of the most challenging questions in multivariable operator theory and functions of several complex variables. However, the research in this direction seems unexplored except the work of Grinshpan, KaliuzhnyiVerbovetskyi, Vinnikov and Woerdeman [14] and the recent paper [5]. Also see Choi and Davidson [6], Drury [12, Holbrook [15, 16], Knese [17] and Kosiński [18] for relevant examples and results.

The complexity of the above problem is further compounded by a number of related (and equally complex) function theoretic problems in several complex variables like commutant lifting theorem, (Toeplitz) corona theorem, Nevanlinna-Pick interpolation theorem, Caratheodory-Fejer theorem and invariant subspace problem. Here we are particularly interested in the commutant lifting theorem, which is also equivalent to the Ando dilation theorem (cf. [13]). The commutant lifting theorem in the setting of scalar-valued Hardy space is due to Sarason [20]. We state here the Sz.-Nagy and Foias version [21] of commutant lifting theorem in the setting of vector-valued Hardy space: Let $\mathcal{E}$ and $\mathcal{E}_{*}$ be Hilbert spaces, $\mathcal{Q} \subseteq H_{\mathcal{E}}^{2}(\mathbb{D})$ and $\mathcal{Q}_{*} \subseteq H_{\mathcal{E}_{*}}^{2}(\mathbb{D})$ be closed subspaces and let $X \in \mathcal{B}\left(\mathcal{Q}, \mathcal{Q}_{*}\right)$. Suppose that $\mathcal{Q}$ and $\mathcal{Q}_{*}$ are shift co-invariant subspaces (invariant under the adjoint of the multiplication operator $M_{z}$ ) of $H_{\mathcal{E}}^{2}(\mathbb{D})$ and $H_{\mathcal{E}_{*}}^{2}(\mathbb{D})$, respectively, and

$$
X\left(\left.P_{\mathcal{Q}} M_{z}\right|_{\mathcal{Q}}\right)=\left(\left.P_{\mathcal{Q}_{*}} M_{z}\right|_{\mathcal{Q}_{*}}\right) X
$$

Then there exists a bounded holomorphic function $\varphi \in H_{\mathcal{B}\left(\mathcal{E}, \mathcal{E}_{*}\right)}^{\infty}(\mathbb{D})$ such that $\|X\|=\|\varphi\|_{\infty}$ (the uniform norm of $\varphi$ over $\mathbb{D}$ ) and

$$
X^{*}=\left.M_{\varphi}^{*}\right|_{\mathcal{Q}_{*}} .
$$

Here and in what follows, $H_{\mathcal{B}\left(\mathcal{E}, \mathcal{E}_{*}\right)}^{\infty}\left(\mathbb{D}^{n}\right)$ denotes the set of all bounded $\mathcal{B}\left(\mathcal{E}, \mathcal{E}_{*}\right)$-valued analytic functions on $\mathbb{D}^{n}$. In connection with the above it is now natural to ask whether the commutant lifting theorem can be extended to the case of vector-valued Hardy space over the polydisc $\mathbb{D}^{n}$ in $\mathbb{C}^{n}$. This has been addressed by Ball, Li, Timotin and Trent [4] for a special class, known as Schur-Agler class, of multipliers. To be more specific, let $\mathcal{E}$ and $\mathcal{E}_{*}$ be Hilbert spaces. The Schur-Agler class $\mathcal{S} \mathcal{A}_{n}\left(\mathcal{E}, \mathcal{E}_{*}\right)$ consists of $\mathcal{B}\left(\mathcal{E}, \mathcal{E}_{*}\right)$-valued analytic functions $\Phi$ on $\mathbb{D}^{n}$ such that $\Phi$ satisfies the $n$-variables von Neumann inequality, that is

$$
\mathcal{S A}_{n}\left(\mathcal{E}, \mathcal{E}_{*}\right)=\left\{\Phi \in H_{\mathcal{B}\left(\mathcal{E}, \mathcal{E}_{*}\right)}^{\infty}\left(\mathbb{D}^{n}\right):\|\Phi(T)\| \leq 1, T \in \mathcal{T}_{1}^{n}(\mathcal{H}) \text { and } \mathcal{H} \text { a Hilbert space }\right\}
$$

where $\mathcal{T}_{1}^{n}(\mathcal{H})=\left\{T \in \mathcal{T}^{n}(\mathcal{H}):\left\|T_{i}\right\|<1, i=1, \ldots, n\right\}$. Here, the functional calculus $\Phi(T)$ is given by

$$
\Phi(T)=S O T-\sum_{\boldsymbol{k} \in \mathbb{Z}_{+}^{n}} \Phi_{\boldsymbol{k}} \otimes T^{\boldsymbol{k}}
$$


where $\Phi(\boldsymbol{z})=\sum_{\boldsymbol{k} \in \mathbb{Z}_{+}^{n}} \Phi_{\boldsymbol{k}} z^{\boldsymbol{k}}$ is the Taylor expansion for $\Phi$ centered at the origin in $\mathbb{C}^{n}$ with $\Phi_{\boldsymbol{k}} \in \mathcal{B}\left(\mathcal{E}, \mathcal{E}_{*}\right)$ and $\boldsymbol{z}^{\boldsymbol{k}}=z_{1}^{k_{1}} \cdots z_{n}^{k_{n}}$ for all $\boldsymbol{k} \in \mathbb{Z}_{+}^{n}$. The elements of $\mathcal{S} \mathcal{A}_{n}\left(\mathcal{E}, \mathcal{E}_{*}\right)$ are called Schur-Agler class functions. It is worth noting that the Schur-Agler class is a proper subset of bounded holomorphic functions in three or more than three variables [6, 15, 16, 17, 18, 22, 23]:

$$
\mathcal{S} \mathcal{A}_{n}\left(\mathcal{E}, \mathcal{E}_{*}\right) \varsubsetneqq H_{\mathcal{B}\left(\mathcal{E}, \mathcal{E}_{*}\right)}^{\infty}\left(\mathbb{D}^{n}\right) \quad(n>2) .
$$

We need one more piece of notation. Given $A \in \mathcal{B}(\mathcal{H})$, the conjugate map 4 ] is the completely positive map $C_{A}: \mathcal{B}(\mathcal{H}) \rightarrow \mathcal{B}(\mathcal{H})$ defined by

$$
C_{A}(X)=A X A^{*} \quad(X \in \mathcal{B}(\mathcal{H})) .
$$

It is easy to see that if $A_{1} A_{2}=A_{2} A_{1}$ for some $A_{1}, A_{2} \in \mathcal{B}(\mathcal{H})$, then $C_{A_{1}} C_{A_{2}}=C_{A_{2}} C_{A_{1}}$.

We are now ready to state the Ball, Li, Timotin and Trent's Schur-Agler class version of commutant lifting theorem (Theorem 2.4 in [4]): Let $\mathcal{Q} \subseteq H_{\mathcal{E}}^{2}\left(\mathbb{D}^{n}\right)$ and $\mathcal{Q}_{*} \subseteq H_{\mathcal{E}_{*}}^{2}\left(\mathbb{D}^{n}\right)$ be closed subspaces and let $X \in \mathcal{B}\left(\mathcal{Q}, \mathcal{Q}_{*}\right)$. Suppose that $M_{z_{i}}^{*} \mathcal{Q} \subseteq \mathcal{Q}$ and $M_{z_{i}}^{*} \mathcal{Q}_{*} \subseteq \mathcal{Q}_{*}$ and

$$
\left(\left.P_{\mathcal{Q}_{*}} M_{z_{i}}\right|_{\mathcal{Q}_{*}}\right) X=X\left(\left.P_{\mathcal{Q}} M_{z_{i}}\right|_{\mathcal{Q}}\right)
$$

for all $i=1, \ldots, n$. Then there exists $\Phi \in \mathcal{S} \mathcal{A}_{n}\left(\mathcal{E}, \mathcal{E}_{*}\right)$ such that $\|X\|=\|\Phi\|_{\infty}$ and

$$
X^{*}=\left.M_{\Phi}^{*}\right|_{\mathcal{Q}_{*}},
$$

if and only if there exist positive operators $G_{i} \in \mathcal{B}\left(\mathcal{Q}_{*}\right), i=1, \ldots, n$, such that

$$
I-X X^{*}=G_{1}+\cdots+G_{n}
$$

and

$$
\left(\prod_{\substack{j=1 \\ j \neq i}}^{n}\left(I_{\mathcal{B}\left(\mathcal{Q}_{*}\right)}-C_{P_{\mathcal{Q}_{*}} M_{z_{i}} \mid \mathcal{Q}_{*}}\right)\right)\left(G_{i}\right) \geq 0,
$$

for all $i=1, \ldots, n$.

Using the above Ball, Li, Timotin and Trent commutant lifting theorem as an inspiration, we introduce a class of operators in $\mathcal{T}^{n}(\mathcal{H})$. But before we do that, we introduce some notation and definitions.

From now on we will assume that $n \geq 3$. For $T \in \mathcal{T}^{n}(\mathcal{H})$ and $1 \leq i \leq n$, define

$$
\hat{T}_{i}=\left(T_{1}, \ldots, T_{i-1}, T_{i+1}, \ldots, T_{n}\right) \in \mathcal{T}^{(n-1)}(\mathcal{H}),
$$

the $(n-1)$-tuple obtained from $T$ by deleting $T_{i}$. Now we define the set

$$
\mathbb{S}_{n}(\mathcal{H})=\left\{T \in \mathcal{T}^{n}(\mathcal{H}): \mathbb{S}_{n}^{-1}\left(T, T^{*}\right) \geq 0 \text { and } T_{i} \text { is pure for all } i=1, \ldots, n\right\}
$$

where

$$
\mathbb{S}_{n}^{-1}\left(T, T^{*}\right)=\sum_{\boldsymbol{k} \in\{0,1\}^{n}}(-1)^{|\boldsymbol{k}|} T^{\boldsymbol{k}} T^{* \boldsymbol{k}}
$$

The elements of $\mathbb{S}_{n}(\mathcal{H})$ are called Szegö n-tuples. Recall that a contraction $X \in \mathcal{T}^{1}(\mathcal{H})$ is said to be pure if $\left\|X^{* m} h\right\| \rightarrow 0$ as $m \rightarrow \infty$ for all $h \in \mathcal{H}$. Now we are ready to define the central object of this paper. 
Definition 1.1. An $n$-tuple $T \in \mathcal{T}^{n}(\mathcal{H})$ is said to be in $\mathcal{P}_{n}(\mathcal{H})$ if $\hat{T}_{n} \in \mathbb{S}_{n-1}(\mathcal{H})$ and there exist positive operators $G_{1}, \ldots, G_{n-1}$ (depending on $T$ ) in $\mathcal{B}(\mathcal{H})$ such that

$$
I-T_{n} T_{n}^{*}=G_{1}+\cdots+G_{n-1}
$$

and

$$
S_{T}\left(G_{i}\right):=\left(\prod_{\substack{j=1 \\ j \neq i}}^{n-1}\left(I_{\mathcal{B}(\mathcal{H})}-C_{T_{j}}\right)\right)\left(G_{i}\right) \geq 0
$$

for all $i=1, \ldots, n-1$.

Definition 1.2. Let $T \in \mathcal{P}_{n}(\mathcal{H})$. We say that $T$ is a finite rank tuple if there exist positive operators $G_{1}, \ldots, G_{n-1}$ associated to $T$ as in the above definition such that

$$
\operatorname{rank}\left(\mathbb{S}_{n-1}^{-1}\left(\hat{T}_{n}, \hat{T}_{n}^{*}\right)\right)<\infty, \quad \text { and } \operatorname{rank}\left(S_{T}\left(G_{i}\right)\right)<\infty,
$$

for all $i=1, \ldots, n-1$.

The main results of this paper says that: If $T \in \mathcal{P}_{n}(\mathcal{H})$ is a finite rank tuple, then

(i) $T$ admits an explicit isometric dilation (see Theorem 4.4), and

(ii) there exists an algebraic variety $V$ in $\overline{\mathbb{D}}^{n}$ such that

$$
\|p(T)\|_{\mathcal{B}(\mathcal{H})} \leq \sup _{\boldsymbol{z} \in V}|p(\boldsymbol{z})|
$$

for all $p \in \mathbb{C}\left[z_{1}, \ldots, z_{n}\right]$ (see Theorem 5.1).

In fact, in Theorem 4.3 we first reprove the Ball, Li, Timotin and Trent commutant lifting theorem. Here, however, with a slightly more elaborated idea we prove an explicit version of the commutant lifting theorem. This method then yields an explicit construction of isometric dilations of finite rank tuples in $\mathcal{T}^{n}(\mathcal{H})$. This is the content of Section 4. Then in Section 5 , as application of the explicit isometric dilations, we prove a refined version of von Neumann inequality, in terms of algebraic varieties in $\overline{\mathbb{D}}^{n}$ (or in $\mathbb{D}^{n}$ ), of finite rank tuples in $\mathcal{P}_{n}(\mathcal{H})$.

In Section 2, we present some elementary examples. In Section 3, we set up some notation and terminology and prove some basic results.

It is worth noting, in this context, that the class of commuting contractions in $\mathcal{P}_{n}(\mathcal{H})$ is larger than the one considered in [5] (see Remark 2.1).

\section{EXAMPLES}

Before we move on to the technical part, we present an elementary but non-trivial example of tuple in $\mathcal{P}_{n}(\mathcal{H})$.

Let $\left(T_{1}, T_{2}\right) \in \mathbb{S}_{2}(\mathcal{H})$ and let $j, k \geq 1$. Suppose $T_{3}=T_{1}^{j} T_{2}^{k}$. Then $T=\left(T_{1}, T_{2}, T_{3}\right) \in \mathcal{P}_{3}(\mathcal{H})$. Indeed, if we set

$$
G_{1}=I-T_{1}^{j} T_{1}^{* j}
$$

and

$$
G_{2}=T_{1}^{j}\left(I-T_{2}^{k} T_{2}^{* k}\right) T_{1}^{* j}
$$

then clearly

$$
I-T_{3} T_{3}^{*}=G_{1}+G_{2} .
$$


On the other hand, $\mathbb{S}_{2}^{-1}\left(\left(T_{1}, T_{2}\right),\left(T_{1}^{*}, T_{2}^{*}\right)\right) \geq 0$ implies that

$$
T_{2}\left(I-T_{1} T_{1}^{*}\right) T_{2}^{*} \leq I-T_{1} T_{1}^{*}
$$

from which it follows that

$$
\begin{aligned}
T_{2} G_{1} T_{2}^{*} & =T_{2}\left(\sum_{i=0}^{j-1} T_{1}^{i}\left(I-T_{1} T_{1}^{*}\right) T_{1}^{* i}\right) T_{2}^{*} \\
& =\sum_{i=0}^{j-1} T_{1}^{i} T_{2}\left(I-T_{1} T_{1}^{*}\right) T_{2}^{*} T_{1}^{* i} \\
& \leq \sum_{i=0}^{j-1} T_{1}^{i}\left(I-T_{1} T_{1}^{*}\right) T_{1}^{* i} \\
& =I-T_{1}^{j} T_{1}^{* j}
\end{aligned}
$$

that is

$$
G_{1}-T_{2} G_{1} T_{2}^{*} \geq 0
$$

Similarly, $T_{1}\left(I-T_{2} T_{2}^{*}\right) T_{1}^{*} \leq I-T_{2} T_{2}^{*}$ implies that

$$
G_{2}-T_{1} G_{2} T_{1}^{*} \geq 0
$$

and hence the claim follows.

In this context we remark, in view of $C_{T_{i}} C_{T_{j}}=C_{T_{j}} C_{T_{i}}$ for all $i=1, \ldots, n$, that (see Definition 1.1)

$$
S_{T}\left(G_{i}\right)=\sum_{\boldsymbol{k} \in \mathbb{Z}_{+}^{n-2}}(-1)^{|\boldsymbol{k}|} \hat{T}_{i, n}^{k} G_{i} \hat{T}_{i, n}^{* \boldsymbol{k}}
$$

where $\hat{T}_{i, n}=\left(T_{1}, \ldots, T_{i-1}, T_{i+1}, \ldots, T_{n-1}\right) \in \mathcal{T}^{(n-2)}(\mathcal{H})$ for $1 \leqslant i \leqslant n-1$. In particular, if $n=4$, then

$$
\prod_{\substack{j=1 \\ j \neq 3}}^{3}\left(I_{\mathcal{B}(\mathcal{H})}-C_{T_{j}}\right)\left(G_{3}\right)=G_{3}-T_{1} G_{3} T_{1}^{*}-T_{2} G_{3} T_{2}^{*}+T_{1} T_{2} G_{3} T_{1}^{*} T_{2}^{*} .
$$

Moreover, $\left(T_{1}, T_{2}, T_{3}\right) \in \mathcal{T}^{3}(\mathcal{H})$ if there exist positive operators $G_{1}$ and $G_{2}$ in $\mathcal{B}(\mathcal{H})$ such that

$$
I-T_{3} T_{3}^{*}=G_{1}+G_{2},
$$

and

$$
G_{2}-T_{1} G_{2} T_{1}^{*} \geq 0 \quad \text { and } \quad G_{1}-T_{2} G_{1} T_{2}^{*} \geq 0 .
$$

Remark 2.1. Let $T=\left(T_{1}, \ldots, T_{n}\right) \in \mathcal{T}^{n}(\mathcal{H})$ and let $1 \leq p<q \leq n$. Recall from Subsection 2.3 in [5] that $T \in \mathcal{T}_{p, q}^{n}(\mathcal{H})$ if $\hat{T}_{p} \in \mathbb{S}_{n-1}(\mathcal{H})$ and $\hat{T}_{q}$ satisfies the Szegö positivity. We claim that

$$
\mathcal{T}_{p, q}^{n}(\mathcal{H}) \subseteq \mathcal{P}_{n}(\mathcal{H})
$$


Indeed, for $T \in \mathcal{T}^{n}(\mathcal{H})$, without any loss of generality, we assume that $\hat{T}_{n} \in \mathbb{S}_{n-1}(\mathcal{H})$ and $\hat{T}_{1}$ satisfies the Szegö positivity. Then we set $G_{1}=I-T_{n} T_{n}^{*}$ and $G_{i}=0$ for all $i=2, \ldots, n-1$, and consequently

$$
\prod_{j=2}^{n-1}\left(I_{\mathcal{B}(\mathcal{H})}-C_{T_{j}}\right)\left(G_{1}\right)=\mathbb{S}_{n-1}^{-1}\left(\hat{T}_{1}, \hat{T}_{1}^{*}\right) \geq 0,
$$

as $\hat{T}_{1} \in \mathbb{S}_{n-1}(\mathcal{H})$. Therefore $T \in \mathcal{P}_{n}(\mathcal{H})$, and hence, $\mathcal{P}_{n}(\mathcal{H})$ is considerably larger than $\mathcal{T}_{p, q}^{n}(\mathcal{H})$.

\section{Preparatory Results}

This section sets up some of the needed terminology and isolates some preparatory results. We start by considering the Hardy space over the unit polydisc.

The Hardy space $H^{2}\left(\mathbb{D}^{n}\right)$ over $\mathbb{D}^{n}$ is the Hilbert space of all analytic functions $f=$ $\sum_{\boldsymbol{k} \in \mathbb{Z}_{+}^{n}} a_{\boldsymbol{k}} z^{k}$ on $\mathbb{D}^{n}$ such that

$$
\|f\|=\left(\sum_{k \in \mathbb{Z}_{+}^{n}}\left|a_{k}\right|^{2}\right)^{\frac{1}{2}}<\infty .
$$

Moreover, for a Hilbert space $\mathcal{E}$, the $\mathcal{E}$-valued Hardy space on $\mathbb{D}^{n}$ is denoted by $H_{\mathcal{E}}^{2}\left(\mathbb{D}^{n}\right)$. We will also identify $H_{\mathcal{E}}^{2}\left(\mathbb{D}^{n}\right)$ with $H^{2}\left(\mathbb{D}^{n}\right) \otimes \mathcal{E}$ via the unitary map $\boldsymbol{z}^{\boldsymbol{k}} \eta \mapsto \boldsymbol{z}^{\boldsymbol{k}} \otimes \eta$ for all $\boldsymbol{k} \in \mathbb{Z}_{+}^{n}$ and $\eta \in \mathcal{E}$. It is well known that the $\mathcal{B}(\mathcal{E})$-valued function

$$
(\boldsymbol{z}, \boldsymbol{w}) \in \mathbb{D}^{n} \times \mathbb{D}^{n} \rightarrow \mathbb{S}_{n}(\boldsymbol{z}, \boldsymbol{w}) I_{\mathcal{E}}
$$

is the reproducing kernel for $H_{\mathcal{E}}^{2}\left(\mathbb{D}^{n}\right)$, where

$$
\mathbb{S}_{n}(\boldsymbol{z}, \boldsymbol{w})=\prod_{i=1}^{n}\left(1-z_{i} \bar{w}_{i}\right)^{-1} \quad\left(\boldsymbol{z}, \boldsymbol{w} \in \mathbb{D}^{n}\right),
$$

is the Szegö kernel on $\mathbb{D}^{n}$. Let $\left(M_{z_{1}}, \ldots, M_{z_{n}}\right)$ denote the $n$-tuple of multiplication operators on $H_{\mathcal{E}}^{2}\left(\mathbb{D}^{n}\right)$. Here $\left(M_{z_{i}} f\right)(\boldsymbol{w}):=w_{i} f(\boldsymbol{w})$ for all $f \in H_{\mathcal{E}}^{2}\left(\mathbb{D}^{n}\right), \boldsymbol{w} \in \mathbb{D}^{n}$ and $i=1, \ldots, n$. It follows from the definition that

$$
M_{z_{i}}^{*} M_{z_{i}}=I_{H_{\mathcal{E}}^{2}\left(\mathbb{D}^{n}\right)}, \quad M_{z_{i}} M_{z_{j}}=M_{z_{j}} M_{z_{i}} \quad \text { and } \quad M_{z_{p}}^{*} M_{z_{q}}=M_{z_{q}} M_{z_{p}}^{*},
$$

for all $i, j=1, \ldots, n$ and $1 \leq p<q \leq n$.

Now we recall the fractional linear transformation representations of Schur-Agler class of functions. Let $\mathcal{H}_{i}, i=1, \ldots, n, \mathcal{E}$ and $\mathcal{E}_{*}$ be Hilbert spaces, and let

$$
E(\boldsymbol{z})=\bigoplus_{i=1}^{n} z_{i} I_{\mathcal{H}_{i}} \in \mathcal{B}\left(\bigoplus_{i=1}^{n} \mathcal{H}_{i}\right),
$$

the diagonal operator for all $\boldsymbol{z} \in \mathbb{D}^{n}$. Let

$$
U=\left[\begin{array}{ll}
A & B \\
C & D
\end{array}\right]: \mathcal{E} \oplus\left(\bigoplus_{i=1}^{n} \mathcal{H}_{i}\right) \rightarrow \mathcal{E}_{*} \oplus\left(\bigoplus_{i=1}^{n} \mathcal{H}_{i}\right),
$$

be a unitary operator (known as colligation matrix). Then the transfer function $\tau_{U}$ corresponding to $U$ is defined by

$$
\tau_{U}(\boldsymbol{z})=A+B E(\boldsymbol{z})\left(I_{\mathcal{H}}-D E(\boldsymbol{z})\right)^{-1} C \quad\left(\boldsymbol{z} \in \mathbb{D}^{n}\right) .
$$


Since $\|D\| \leq 1$, and so $\|D E(\boldsymbol{z})\|<1$ for all $\boldsymbol{z} \in \mathbb{D}^{n}$, it follows that $\tau_{U}$ is a $\mathcal{B}\left(\mathcal{E}, \mathcal{E}_{*}\right)$-valued analytic function on $\mathbb{D}^{n}$. Moreover, a standard and well-known computation (cf. [2], 44]) implies that

$$
I-\tau_{U}(\boldsymbol{z})^{*} \tau_{U}(\boldsymbol{z})=C^{*}\left(I_{\mathcal{H}}-E(\boldsymbol{z})^{*} D^{*}\right)^{-1}\left(I_{\mathcal{H}}-E(\boldsymbol{z})^{*} E(\boldsymbol{z})\right)\left(I_{\mathcal{H}}-D E(\boldsymbol{z})\right)^{-1} C,
$$

for all $z \in \mathbb{D}^{n}$. We conclude that $\tau_{U}$ is a contractive multiplier, that is, $\tau_{U} \in H_{\mathcal{B}\left(\mathcal{E}, \mathcal{E}_{*}\right)}^{\infty}\left(\mathbb{D}^{n}\right)$ and $\left\|M_{\tau_{U}}\right\| \leq 1$ where $M_{\tau_{U}}: H_{\mathcal{E}}^{2}\left(\mathbb{D}^{n}\right) \rightarrow H_{\mathcal{E}_{*}}^{2}\left(\mathbb{D}^{n}\right)$ is the multiplication operator defined by

$$
M_{\tau_{U}} f=\tau_{U} f \quad\left(f \in H_{\mathcal{E}}^{2}\left(\mathbb{D}^{n}\right)\right) .
$$

The celebrated realization theorem of Agler states the following: $\Phi \in \mathcal{S} \mathcal{A}_{n}\left(\mathcal{E}, \mathcal{E}_{*}\right)$ (see Section 1 for the definition of $\left.\mathcal{S} \mathcal{A}_{n}\left(\mathcal{E}, \mathcal{E}_{*}\right)\right)$ if and only if there exist Hilbert spaces $\left\{\mathcal{H}_{i}\right\}_{i=1}^{n}$ and a unitary colligation matrix $U$, as in (3.1), such that $\Phi=\tau_{U}$.

The following basic result will be useful. Part of the proof relies on the following general result: Let $A$ be a contraction on a Hilbert space $\mathcal{K}, \lambda \in \mathbb{T}$ and let $f \in \mathcal{K}$. Then

$$
A f=\lambda f \Leftrightarrow A^{*} f=\bar{\lambda} f .
$$

Lemma 3.1. Let $\mathcal{H}_{1}, \ldots, \mathcal{H}_{n}$ and $\mathcal{E}$ be finite dimensional Hilbert spaces, and let

$$
U=\left[\begin{array}{ll}
A & B \\
C & D
\end{array}\right] \in \mathcal{B}\left(\mathcal{E} \oplus\left(\bigoplus_{i=1}^{n} \mathcal{H}_{i}\right)\right),
$$

be a unitary matrix. Then the transfer function $\tau_{U}$ defined by

$$
\tau_{U}(\boldsymbol{z})=A+B E(\boldsymbol{z})\left(I_{\mathcal{H}}-D E(\boldsymbol{z})\right)^{-1} C \quad\left(\boldsymbol{z} \in \mathbb{D}^{n}\right),
$$

is unitary-valued a.e. on $\mathbb{T}^{n}$. Moreover, if $A \in \mathcal{B}(\mathcal{E})$ is a completely non-unitary, then for each $\boldsymbol{z} \in \mathbb{D}^{n}$ the operator $\tau_{U}(\boldsymbol{z})$ does not have eigenvalues on the unit circle.

Proof. Clearly, $\boldsymbol{z} \mapsto \operatorname{det}\left(I_{\mathcal{H}}-D E(\boldsymbol{z})\right)$ is a non-vanishing polynomial in $\mathbb{D}^{n}$ and hence

$$
\boldsymbol{z} \mapsto\left(I_{\mathcal{H}}-D E(\boldsymbol{z})\right)^{-1}
$$

is a non-zero rational function in $H^{\infty}\left(\mathbb{D}^{n}\right)$. This means that $\boldsymbol{z} \mapsto \operatorname{det}\left(I_{\mathcal{H}}-D E(\boldsymbol{z})\right)$ does not vanish on a set of positive measure on $\mathbb{T}^{n}$ and hence $\boldsymbol{z} \mapsto(I-D E(\boldsymbol{z}))^{-1}$ exists a.e. on $\mathbb{T}^{n}$. The first part now follows from (3.3).

For the second part, let $\boldsymbol{z} \in \mathbb{D}^{n}, \lambda \in \mathbb{T}$ and let $f$ be a non-zero element in $\mathcal{E}$. Suppose

$$
\tau_{U}(\boldsymbol{z}) f=\lambda f .
$$

Since $\tau_{U}(\boldsymbol{z})$ is a contraction, it follows that

$$
\tau_{U}(\boldsymbol{z})^{*} f=\bar{\lambda} f
$$

and so

$$
\left(I_{\mathcal{E}}-\tau_{U}(\boldsymbol{z})^{*} \tau_{U}(\boldsymbol{z})\right) f=0 .
$$

It follows easily from (3.3) that $C f=0$. Then $A f=\tau_{U}(\boldsymbol{z}) f=\lambda f$ and so

$$
A^{*} f=\bar{\lambda} f \text {. }
$$

This implies that $A$ has a non-trivial unitary part, which leads to a contradiction. 
Let us now proceed to set the stage of the technical part in the proofs of our main results. Let $\mathcal{E}$ be a Hilbert space. Define the evaluation map $e v_{0}: H_{\mathcal{E}}^{2}\left(\mathbb{D}^{n}\right) \rightarrow \mathcal{E}$ by

$$
e v_{0} f=f(\mathbf{0}) \quad\left(f \in H_{\mathcal{E}}^{2}\left(\mathbb{D}^{n}\right)\right) .
$$

Then $e v_{0}^{*}: \mathcal{E} \rightarrow H_{\mathcal{E}}^{2}\left(\mathbb{D}^{n}\right)$ sends $\eta \in \mathcal{E}$ to the constant function $\eta \in H_{\mathcal{E}}^{2}\left(\mathbb{D}^{n}\right)$ in the following sense:

$$
\left(e v_{0}^{*} \eta\right)(\boldsymbol{z})=\eta \quad\left(\boldsymbol{z} \in \mathbb{D}^{n}\right) .
$$

Now let $T \in \mathcal{T}^{n}(\mathcal{H})$ and suppose $\hat{T}_{n} \in \mathbb{S}_{n-1}(\mathcal{H})$. Let $\left\{F_{1}, \ldots, F_{n-1}\right\} \subseteq \mathcal{B}(\mathcal{H})$ and $\mathcal{K}$ be a Hilbert space. For each $i=1, \ldots, n-1$, set

$$
\mathcal{F}_{i}=\overline{\operatorname{ran}} F_{i}
$$

and define

$$
\mathcal{F}=\left(\bigoplus_{i=1}^{n-2} \mathcal{F}_{i}\right) \oplus\left(\mathcal{F}_{n-1} \oplus \mathcal{K}\right),
$$

the $(n-1)$-fold direct sum of Hilbert spaces. Define

$$
D_{\hat{T}_{n}}=\left(\mathbb{S}_{n-1}^{-1}\left(\hat{T}_{n}, \hat{T}_{n}^{*}\right)\right)^{\frac{1}{2}} \quad \text { and } \quad D_{\hat{T}_{n}}=\overline{\operatorname{ran}} D_{\hat{T}_{n}}
$$

Let

$$
U=\left[\begin{array}{cc}
A & B \\
C & D
\end{array}\right]: \mathcal{D}_{\hat{T}_{n}} \oplus \mathcal{F} \rightarrow \mathcal{D}_{\hat{T}_{n}} \oplus \mathcal{F},
$$

be a unitary operator such that

$$
U\left(D_{\hat{T}_{n}} h, F_{1} T_{1}^{*} h, \ldots, F_{n-2} T_{n-2}^{*} h,\left(F_{n-1} T_{n-1}^{*} h, 0_{\mathcal{K}}\right)\right)=\left(D_{\hat{T}_{n}} T_{n}^{*} h, F_{1} h, \ldots, F_{n-2} h,\left(F_{n-1} h, 0_{\mathcal{K}}\right)\right),
$$

for all $h \in \mathcal{H}$. Define $\imath$ and $Y$ in $\mathcal{B}(\mathcal{H}, \mathcal{F})$ by

$$
\imath h=\left(F_{1} h, \ldots, F_{n-2} h,\left(F_{n-1} h, 0_{\mathcal{K}}\right)\right),
$$

and

$$
Y h=\left(F_{1} T_{1}^{*} h, \ldots, F_{n-2} T_{n-2}^{*} h,\left(F_{n-1} T_{n-1}^{*} h, 0_{\mathcal{K}}\right)\right),
$$

for all $h \in \mathcal{H}$. Then (3.5) yields

$$
\left[\begin{array}{cc}
A & B \\
C & D
\end{array}\right]\left[\begin{array}{c}
D_{\hat{T}_{n}} h \\
Y h
\end{array}\right]=\left[\begin{array}{c}
D_{\hat{T}_{n}} T_{n}^{*} h \\
\imath h
\end{array}\right]
$$

Therefore

$$
D_{\hat{T}_{n}} T_{n}^{*}=A D_{\hat{T}_{n}}+B Y \quad \text { and } \quad \imath=C D_{\hat{T}_{n}}+D Y .
$$

Suppose $\Phi=\tau_{U^{*}}$, that is (see (3.2))

$$
\Phi(\boldsymbol{z})=A^{*}+C^{*}\left(I_{\mathcal{F}}-E(\boldsymbol{z}) D^{*}\right)^{-1} E(\boldsymbol{z}) B^{*} \quad\left(\boldsymbol{z} \in \mathbb{D}^{n-1}\right) .
$$

Define $\Psi: \mathcal{F} \rightarrow H_{\mathcal{D}_{\hat{T}_{n}}}^{2}\left(\mathbb{D}^{n-1}\right)$ by

$$
[\Psi x](\boldsymbol{z})=C^{*}\left(I_{\mathcal{F}}-E(z) D^{*}\right)^{-1} x \quad\left(x \in \mathcal{F}, \boldsymbol{z} \in \mathbb{D}^{n-1}\right),
$$


and set

$$
\Psi=\left[\Psi_{1} \ldots \Psi_{n-1}\right]
$$

where, $\Psi_{i}: \mathcal{F}_{i} \rightarrow H_{\mathcal{D}_{\hat{T}_{n}}}^{2}\left(\mathbb{D}^{n-1}\right)$ for all $i=1, \ldots, n-2$, and $\Psi_{n-1}: \mathcal{F}_{n-1} \oplus \mathcal{K} \rightarrow H_{\mathcal{D}_{\hat{T}_{n}}}^{2}\left(\mathbb{D}^{n-1}\right)$. It is convenient to represent $D$ and $B$ as row operators:

$$
D=\left[\begin{array}{llll}
D_{1} & \cdots & D_{n-2} & D_{n-1}
\end{array}\right]:\left(\bigoplus_{i=1}^{n-2} \mathcal{F}_{i}\right) \oplus\left(\mathcal{F}_{n-1} \oplus \mathcal{K}\right) \rightarrow \mathcal{F}
$$

where $D_{i}=\left.D\right|_{\mathcal{F}_{i}}: \mathcal{F}_{i} \rightarrow \mathcal{F}$ for all $i=1, \ldots, n-2$ and $D_{n-1}=\left.D\right|_{\mathcal{F}_{n-1} \oplus \mathcal{K}}$, and similarly

$$
B=\left[\begin{array}{llll}
B_{1} & \cdots & B_{n-2} & B_{n-1}
\end{array}\right]:\left(\bigoplus_{i=1}^{n-2} \mathcal{F}_{i}\right) \oplus\left(\mathcal{F}_{n-1} \oplus \mathcal{K}\right) \rightarrow \mathcal{D}_{\hat{T}_{n}}
$$

The following two lemmas will be used in Section 4 to prove the dilation theorem. We closely follow the ideas of [4].

Lemma 3.2. Let $\Phi$ and $\Psi$ be as above. Then $\Psi$ is a contraction. Moreover

$$
\Psi=e v_{0}^{*} C^{*}+\sum_{i=1}^{n-1} M_{z_{j}} \Psi_{j} D_{j}^{*}
$$

and

$$
M_{\Phi} e v_{0}^{*}=e v_{0}^{*} A^{*}+\sum_{j=1}^{n-1} M_{z_{j}} \Psi_{j} B_{j}^{*}
$$

Proof. It follows directly from Lemma 3.2 of [4] that $\Psi$ is a contraction. For the second part, let $x=\left(x_{1}, \ldots, x_{n-1}\right) \in \mathcal{F}$ (note that $\left.x_{n-1} \in \mathcal{F}_{n-1} \oplus \mathcal{K}\right)$ and $\boldsymbol{z}=\left(z_{1}, \ldots, z_{n-1}\right) \in \mathbb{D}^{n-1}$. Then

$$
\Psi E(\boldsymbol{z}) x=\sum_{j=1}^{n-1} \Psi_{j} z_{j} x_{j}=\sum_{j=1}^{n-1} z_{j} \Psi_{j} x_{j}
$$


and hence

$$
\begin{aligned}
{[\Psi x](\boldsymbol{z}) } & =C^{*}\left(I-E(z) D^{*}\right)^{-1} x \\
& =C^{*}\left(I-E(\boldsymbol{z}) D^{*}\right)^{-1}\left(I-E(\boldsymbol{z}) D^{*}+E(\boldsymbol{z}) D^{*}\right) x \\
& =C^{*} x+\left(C^{*}\left(I-E(\boldsymbol{z}) D^{*}\right)^{-1}\right)\left(E(\boldsymbol{z}) D^{*} x\right) \\
& =\left(e v_{0}^{*} C^{*} x\right)(\boldsymbol{z})+\left[\Psi E(\boldsymbol{z}) D^{*} x\right](\boldsymbol{z}) \\
& =\left(e v_{0}^{*} C^{*} x\right)(\boldsymbol{z})+\left(\sum_{j=1}^{n-1} z_{j} \Psi_{j} D_{j}^{*} x\right)(\boldsymbol{z}) \\
& =\left(e v_{0}^{*} C^{*} x\right)(\boldsymbol{z})+\sum_{j=1}^{n-1} z_{j}\left(\Psi_{j} D_{j}^{*} x\right)(\boldsymbol{z}) \\
& =\left(e v_{0}^{*} C^{*} x\right)(\boldsymbol{z})+\sum_{j=1}^{n-1}\left(M_{z_{j}} \Psi_{j} D_{j}^{*} x\right)(\boldsymbol{z}) .
\end{aligned}
$$

This implies that $\Psi=e_{0}^{*} C^{*}+\sum_{i=1}^{n-1} M_{z_{j}} \Psi_{j} D_{j}^{*}$. On the other hand, for each $\eta \in \mathcal{D}_{\hat{T}_{n}}$, we have

$$
M_{\Phi} e v_{0}^{*} \eta=e v_{0}^{*} A^{*} \eta+\Psi E(\boldsymbol{z}) B^{*} \eta=e v_{0}^{*} A^{*} \eta+\left[\sum_{j=1}^{n-1} M_{z_{j}} \Psi_{j} B_{j}^{*}\right](\eta) .
$$

This completes the proof.

For each $X \in \mathcal{B}(\mathcal{H})$ and natural number $m$ we define (see page 656 in [4]) a positive map

$$
\Sigma_{X}^{m}: \mathcal{B}(\mathcal{H}) \rightarrow \mathcal{B}(\mathcal{H})
$$

as

$$
\Sigma_{X}^{m}(A)=\sum_{k=0}^{m-1} X^{k} A X^{* k} \quad(A \in \mathcal{B}(\mathcal{H})) .
$$

Let $T=\left(T_{1}, \ldots, T_{n}\right)$ be an $n$-tuple of commuting operators on $\mathcal{H}$. It is easy to see that

$$
\Sigma_{T_{i}}^{m} \Sigma_{T_{j}}^{m}=\Sigma_{T_{j}}^{m} \Sigma_{T_{i}}^{m},
$$

for all $i, j=1, \ldots, n$. Define

$$
\Sigma_{T}^{m}(A)=\left(\prod_{j=1}^{n} \Sigma_{T_{j}}^{m}\right)(A) \quad(A \in \mathcal{B}(\mathcal{H})) .
$$

Clearly, if $A \geq 0$, then $\left\{\Sigma_{T}^{m}(A)\right\}_{m=1}^{\infty}$ is an increasing sequence of positive operators and we set

$$
\Sigma_{T}(A)=S O T-\lim _{m \rightarrow \infty} \Sigma_{T}^{m}(A),
$$

provided the limit exists. Moreover, for each $i, j=1, \ldots, n$, and $m \geq 1$, we have

$$
\Sigma_{T_{i}}^{m} C_{T_{j}}=C_{T_{j}} \Sigma_{T_{i}}^{m},
$$


as $T$ is a commuting tuple (see (1.1) for the definition of the conjugate map $C_{T_{j}}$ ). Therefore

$$
\Sigma_{T_{i}}^{m}\left(I-C_{T_{i}}\right)=\left(I-C_{T_{i}}\right) \Sigma_{T_{i}}^{m}=\left(I-C_{T_{i}^{m}}\right),
$$

for all $i=1, \ldots, n$, and hence

$$
\Sigma_{T}^{m}\left(\prod_{j=1}^{n}\left(I_{\mathcal{B}(\mathcal{H})}-C_{T_{j}}\right)\right)=\left(\prod_{j=1}^{n}\left(I_{\mathcal{B}(\mathcal{H})}-C_{T_{j}^{m}}\right)\right) .
$$

Finally, it will be clear from the context in what follows that given a Hilbert space $\mathcal{E}, M_{z}$ will denote the $n$-tuple of multiplication operators $\left(M_{z_{1}}, \ldots, M_{z_{n}}\right)$ on $H_{\mathcal{E}}^{2}\left(\mathbb{D}^{n}\right)$. Consequently, $\hat{M}_{z_{j}}$ will denote the $(n-1)$-tuple

$$
\left(M_{z_{1}}, \ldots, M_{z_{j-1}}, M_{z_{j+1}}, \ldots, M_{z_{n}}\right),
$$

obtained from $M_{z}$ by removing $M_{z_{j}}$.

We now prove a technical lemma.

Lemma 3.3. Let $\mathcal{H}_{1}, \ldots, \mathcal{H}_{n}$ and $\mathcal{E}$ be Hilbert spaces, $\Psi=\left[\Psi_{1} \cdots \Psi_{n}\right]: \oplus_{i=1}^{n} \mathcal{H}_{i} \rightarrow H_{\mathcal{E}}^{2}\left(\mathbb{D}^{n}\right)$ a bounded linear operator and let $\Phi \in H_{\mathcal{B}(\mathcal{E})}^{\infty}\left(\mathbb{D}^{n}\right)$. If

$$
\left\|\left(e v_{0} f, \Psi_{1}^{*} M_{z_{1}}^{*} f, \ldots, \Psi_{n}^{*} M_{z_{n}}^{*} f\right)\right\|_{\mathcal{E} \oplus\left(\oplus_{i=1}^{n} \mathcal{H}_{i}\right)}=\left\|\left(e v_{0} M_{\Phi}^{*} f, \Psi_{1}^{*} f, \ldots, \Psi_{n}^{*} f\right)\right\|_{\mathcal{E} \oplus\left(\oplus_{i=1}^{n} \mathcal{H}_{i}\right)},
$$

for all $f \in H_{\mathcal{E}}^{2}\left(\mathbb{D}^{n}\right)$, then

$$
\Sigma_{\hat{M}_{z_{j}}}\left(\Psi_{j} \Psi_{j}^{*}\right)<\infty
$$

for all $j=1, \ldots, n$.

Proof. The norm equality condition implies that

$$
e v_{0}^{*} e v_{0}+\sum_{j=1}^{n} M_{z_{j}} \Psi_{j} \Psi_{j}^{*} M_{z_{j}}^{*}=M_{\Phi} e_{0}^{*} e_{0} M_{\Phi}^{*}+\sum_{j=1}^{n} \Psi_{j} \Psi_{j}^{*},
$$

and so

$$
\begin{aligned}
\sum_{j=1}^{n} \Psi_{j} \Psi_{j}^{*}-\sum_{j=1}^{n} M_{z_{j}} \Psi_{j} \Psi_{j}^{*} M_{z_{j}}^{*} & =e v_{0}^{*} e v_{0}-M_{\Phi} e v_{0}^{*} e v_{0} M_{\Phi}^{*} \\
& =\left(\prod_{j=1}^{n}\left(I-C_{M_{z_{j}}}\right)\right)(I)-M_{\Phi}\left(\left(\prod_{j=1}^{n}\left(I-C_{M_{z_{j}}}\right)\right)(I)\right) M_{\Phi}^{*} .
\end{aligned}
$$

This gives

$$
\sum_{j=1}^{n}\left(I-C_{M_{z_{j}}}\right)\left(\Psi_{j} \Psi_{j}^{*}\right)=\left(\prod_{j=1}^{n}\left(I-C_{M_{z_{j}}}\right)\right)\left(I-M_{\Phi} M_{\Phi}^{*}\right),
$$

as $M_{\Phi} M_{z_{i}}=M_{z_{i}} M_{\Phi}$ for all $i=1, \ldots, n$. Applying $\Sigma_{M_{z}}^{m}$ to both sides of the above equality and then using (3.11) and (3.12) we obtain

$$
\sum_{j=1}^{n} \Sigma_{\hat{M}_{z_{j}}}^{m}\left(\left(I-C_{M_{z_{j}}^{m}}\right)\left(\Psi_{j} \Psi_{j}^{*}\right)\right)=\prod_{j=1}^{n}\left(I-C_{M_{z_{j}}^{m}}\right)\left(I-M_{\Phi} M_{\Phi}^{*}\right) .
$$


If we set

$$
\mathcal{K}_{0}=\bigcup_{k=0}^{\infty} \bigcap_{j=1}^{n} \operatorname{Ker} M_{z_{j}}^{* k}
$$

then for any $f \in \mathcal{K}_{0}$ we have

$$
\sum_{j=1}^{n} \sum_{\hat{M}_{z_{j}}}^{m}\left(\Psi_{j} \Psi_{j}^{*}\right) f=\left(I-M_{\Phi} M_{\Phi}^{*}\right) f
$$

for sufficiently large $m$. Since $\mathcal{K}_{0}$ is dense in $H_{\mathcal{E}}^{2}\left(\mathbb{D}^{n}\right)$, it follows that $\Sigma_{\hat{M}_{z_{j}}}\left(\Psi_{j} \Psi_{j}^{*}\right)<\infty$ for all $j=1, \ldots, n$.

\section{Dilations of tuples in $\mathcal{P}_{n}(\mathcal{H})$}

The purpose of this section is to construct explicit liftings and isometric dilations of finite rank tuples in $\mathcal{P}_{n}(\mathcal{H})$. Note that if $V \in \mathcal{T}^{n}(\mathcal{K})$ is an isometric dilation of $T \in \mathcal{T}^{n}(\mathcal{H})$ with $\Pi: \mathcal{H} \rightarrow \mathcal{K}$ as the corresponding intertwining isometry (see the definition of dilations in Section 1) and

$$
\mathcal{Q}=\operatorname{ran} \Pi \text {, }
$$

then $\mathcal{Q}$ is a joint invariant subspace for $\left(V_{1}^{*}, \ldots, V_{n}^{*}\right)$. Moreover, $\left(T_{1}, \ldots, T_{n}\right)$ on $\mathcal{H}$ and $\left(\left.P_{\mathcal{Q}} V_{1}\right|_{\mathcal{Q}}, \ldots,\left.P_{\mathcal{Q}} V_{n}\right|_{\mathcal{Q}}\right)$ on $\mathcal{Q}$ are unitarily equivalent (via the unitary $\Pi: \mathcal{H} \rightarrow \mathcal{Q}$ ), and

$$
\left(\left.P_{\mathcal{Q}} V\right|_{\mathcal{Q}}\right)^{* \boldsymbol{k}}=\left.V^{* \boldsymbol{k}}\right|_{\mathcal{Q}}
$$

for all $k \in \mathbb{Z}_{+}^{n}$.

The following dilation result is well known (see [8], [19] and also see [5]):

Theorem 4.1. Let $T \in \mathbb{S}_{n}(\mathcal{H})$. Suppose $D_{T}=\mathbb{S}_{n}^{-1}\left(T, T^{*}\right)^{1 / 2}$ and $\mathcal{D}_{T}=\overline{\operatorname{ran}} D_{T}$. Then $\Pi: \mathcal{H} \rightarrow H_{\mathcal{D}_{T}}^{2}\left(\mathbb{D}^{n}\right)$, defined by

$$
(\Pi h)(\boldsymbol{z})=\sum_{\boldsymbol{k} \in \mathbb{Z}_{+}^{n}} \boldsymbol{z}^{\boldsymbol{k}} D_{T} T^{* \boldsymbol{k}} h \quad\left(h \in \mathcal{H}, \boldsymbol{z} \in \mathbb{D}^{n}\right),
$$

is an isometry and satisfies $\Pi T_{i}^{*}=M_{z_{i}}^{*} \Pi$ for all $i=1, \ldots, n$. Moreover, the dilation is minimal, that is

$$
H_{\mathcal{D}_{T}}^{2}\left(\mathbb{D}^{n}\right)=\overline{\operatorname{span}}\left\{\boldsymbol{z}^{\boldsymbol{k}}(\Pi \mathcal{H}): \boldsymbol{k} \in \mathbb{Z}_{+}^{n}\right\}
$$

We call the map $\Pi$ as the canonical dilation of $T$. The following standard lemma will be useful.

Lemma 4.2. Let $T \in \mathbb{S}_{n}(\mathcal{H})$ and let $\Pi$ be the canonical dilation of $T$. Then for all $\boldsymbol{k} \in \mathbb{Z}_{+}^{n}$ and $\eta \in \mathcal{D}_{T}$, we have

$$
\Pi^{*}\left(z^{k} \otimes \eta\right)=T^{k} D_{T} \eta
$$

Proof. For $h \in \mathcal{H}$, it is easy to see that

$$
e v_{0} \Pi h=e v_{0}\left(\sum_{l \in \mathbb{Z}_{+}^{n-1}} z^{l} \otimes D_{T} T^{* l} h\right)=D_{T} h
$$


thus

$$
e v_{0} \Pi=D_{T}
$$

Hence, for $\eta \in \mathcal{D}_{T}$ and $\boldsymbol{k} \in \mathbb{Z}_{+}^{n-1}$ we obtain

$$
\Pi^{*}\left(\boldsymbol{z}^{\boldsymbol{k}} \otimes \eta\right)=\Pi^{*} M_{z}^{k} e v_{0}^{*} \eta=T^{k} \Pi^{*} e v_{0}^{*} \eta=T^{k} D_{T} \eta,
$$

and the proof follows.

We are now ready to prove the first commutant lifting theorem of this paper. The explicit representation of the lifting $\Phi \in \mathcal{S} \mathcal{A}_{n-1}\left(\mathcal{D}_{\hat{T}_{n}}, \mathcal{D}_{\hat{T}_{n}}\right)$ below will be useful in our isometric dilations and von Neumann inequality. For a $T \in \mathcal{T}^{n}(\mathcal{H})$ and $1 \leq j \leq n-1$, recall that, $\hat{T}_{j, n}$ denotes the $(n-2)$-tuple

$$
\left(T_{1}, \ldots, T_{j-1}, T_{j+1}, \ldots, T_{n-1}\right),
$$

obtained from $T$ by removing $T_{j}$ and $T_{n}$

Theorem 4.3. Let $\mathcal{H}$ and $\mathcal{K}$ be Hilbert spaces, $T \in \mathcal{T}^{n}(\mathcal{H})$, and let $\left\{F_{1}, \ldots, F_{n-1}\right\} \subseteq \mathcal{B}(\mathcal{H})$. Suppose $\hat{T}_{n} \in \mathbb{S}_{n-1}(\mathcal{H}), \mathcal{F}_{i}=\overline{\operatorname{ran}} F_{i}$ for all $i=1, \ldots, n-1, \Pi: \mathcal{H} \rightarrow H_{\mathcal{D}_{\hat{T}_{n}}}^{2}\left(\mathbb{D}^{n-1}\right)$ is the canonical dilation of $\hat{T}_{n}$, and

$$
\mathcal{F}=\left(\bigoplus_{i=1}^{n-2} \mathcal{F}_{i}\right) \oplus\left(\mathcal{F}_{n-1} \oplus \mathcal{K}\right)
$$

If $\Sigma_{\hat{T}_{i, n}}\left(F_{i}^{*} F_{i}\right)$ exists for all $i=1, \ldots, n-1$, and $U: \mathcal{D}_{\hat{T}_{n}} \oplus \mathcal{F} \rightarrow \mathcal{D}_{\hat{T}_{n}} \oplus \mathcal{F}$ is a unitary satisfying

$$
U\left(D_{\hat{T}_{n}} h, F_{1} T_{1}^{*} h, \ldots, F_{n-2} T_{n-2}^{*} h,\left(F_{n-1} T_{n-1}^{*} h, 0_{\mathcal{K}}\right)\right)=\left(D_{\hat{T}_{n}} T_{n}^{*} h, F_{1} h, \ldots, F_{n-2} h,\left(F_{n-1} h, 0_{\mathcal{K}}\right)\right),
$$

for all $h \in \mathcal{H}$, then

$$
\Pi T_{n}^{*}=M_{\Phi}^{*} \Pi,
$$

where $\Phi \in \mathcal{S} \mathcal{A}_{n-1}\left(\mathcal{D}_{\hat{T}_{n}}, \mathcal{D}_{\hat{T}_{n}}\right)$ is the transfer function of $U^{*}$.

Proof. Let $U=\left[\begin{array}{cc}A & B \\ C & D\end{array}\right]$ be the block matrix representation of $U$ with respect to the decomposition $D_{\hat{T}_{n}} \oplus \mathcal{F}$. Suppose $\Phi=\tau_{U^{*}}($ see $(3.9)), \Psi$ be as in $(3.10)$ and let $f \in H_{\mathcal{D}_{\hat{T}_{n}}}^{2}\left(\mathbb{D}^{n-1}\right)$. Then, in view of (3.5), Lemma 3.2 implies that

$$
U\left(e v_{0} f, \Psi_{1}^{*} M_{z_{1}}^{*} f, \ldots, \Psi_{n-1}^{*} M_{z_{n-1}}^{*} f\right)=\left(e v_{0} M_{\Phi}^{*} f, \Psi_{1}^{*} f, \ldots, \Psi_{n-1}^{*} f\right) .
$$

In particular

$$
\left\|\left(e v_{0} f, \Psi_{1}^{*} M_{z_{1}}^{*} f, \ldots, \Psi_{n-1}^{*} M_{z_{n-1}}^{*} f\right)\right\|=\left\|\left(e v_{0} M_{\Phi}^{*} f, \Psi_{1}^{*} f, \ldots, \Psi_{n-1}^{*} f\right)\right\|,
$$

and therefore

$$
\Sigma_{\hat{M}_{z_{j}}}\left(\Psi_{j} \Psi_{j}^{*}\right)<\infty,
$$

for all $j=1, \ldots, n-1$, by Lemma 3.3. Hence

$$
\Sigma_{\hat{T}_{j, n}}\left(\Pi^{*} \Psi_{j} \Psi_{j}^{*} \Pi\right)=\Pi^{*}\left(\Sigma_{\hat{M}_{z_{j}}}\left(\Psi_{j} \Psi_{j}^{*}\right)\right) \Pi<\infty,
$$


for all $j=1, \ldots, n-1$. Now define $\imath$ and $Y$ in $\mathcal{B}(\mathcal{H}, \mathcal{F})$ as (see (3.6) and (3.7))

$$
\imath h=\left(F_{1} h, \ldots, F_{n-2} h,\left(F_{n-1} h, 0_{\mathcal{K}}\right)\right),
$$

and

$$
Y h=\left(F_{1} T_{1}^{*} h, \ldots, F_{n-2} T_{n-2}^{*} h,\left(F_{n-1} T_{n-1}^{*} h, 0_{\mathcal{K}}\right)\right),
$$

for all $h \in \mathcal{H}$. Also define $\Gamma: \mathcal{F} \rightarrow \mathcal{H}$ by

$$
\Gamma=\imath^{*}-\Pi^{*} \Psi
$$

We set $\Gamma=\left[\Gamma_{1} \cdots \Gamma_{n-1}\right]$, where

$$
\Gamma_{i}=\left.\Gamma\right|_{\mathcal{F}_{i}}=\left.F_{i}^{*}\right|_{\mathcal{F}_{i}}-\Pi^{*} \Psi_{i}: \mathcal{F}_{i} \rightarrow \mathcal{H}
$$

for all $i=1, \ldots, n-2$, and

$$
\Gamma_{n-1}=\left.\Gamma\right|_{\mathcal{F}_{n-1} \oplus \mathcal{K}}=\left[\begin{array}{ll}
\left.F_{n-1}^{*}\right|_{\mathcal{F}_{n-1}} & 0
\end{array}\right]-\Pi^{*} \Psi_{n-1}: \mathcal{F}_{n-1} \oplus \mathcal{K} \rightarrow \mathcal{H}
$$

Now for each $j=1, \ldots, n-2$, we have

$$
\begin{aligned}
\Gamma_{j} \Gamma_{j}^{*} & =\left(\left.F_{j}^{*}\right|_{\mathcal{F}_{j}}-\Pi^{*} \Psi_{j}\right)\left(F_{j}-\Psi_{j}^{*} \Pi\right) \\
& \leq\left(\left.F_{j}^{*}\right|_{\mathcal{F}_{j}}-\Pi^{*} \Psi_{j}\right)\left(F_{j}-\Psi_{j}^{*} \Pi\right)+\left(\left.F_{j}^{*}\right|_{\mathcal{F}_{j}}+\Pi^{*} \Psi_{j}\right)\left(F_{j}+\Psi_{j}^{*} \Pi\right) \\
& =2\left(F_{j}^{*} F_{j}+\Pi^{*} \Psi_{j} \Psi_{j}^{*} \Pi\right),
\end{aligned}
$$

and hence

$$
\Sigma_{\hat{T}_{j, n}}\left(\Gamma_{j} \Gamma_{j}^{*}\right)<\infty
$$

By a similar computation, we also have that $\Sigma_{\hat{T}_{n-1, n}}\left(\Gamma_{j} \Gamma_{j}^{*}\right)<\infty$. On the other hand, since $(\operatorname{see}(3.8))$

$$
\imath=C D_{\hat{T}_{n}}+D Y,
$$

we have

$$
\begin{aligned}
\Gamma & =\imath^{*}-\Pi^{*} \Psi \\
& =D_{\hat{T}_{n}} C^{*}+Y^{*} D^{*}-\Pi^{*} Q C^{*}-\Pi^{*} \sum_{j=1}^{n-1} M_{z_{j}} \Psi_{j} D_{j}^{*} \\
& =D_{\hat{T}_{n}} C^{*}+\sum_{j=1}^{n-1} T_{j} F_{j}^{*} D_{j}^{*}-D_{\hat{T}_{n}} C^{*}-\sum_{j=1}^{n-1} T_{j} \Pi^{*} \Psi_{j} D_{j}^{*} \\
& =\sum_{j=1}^{n-1} T_{j} \Gamma_{j} D_{j}^{*} \\
& =\left[\begin{array}{lll}
T_{1} \Gamma_{1} & \cdots & T_{n-1} \Gamma_{n-1}
\end{array}\right]\left[\begin{array}{c}
D_{1}^{*} \\
\vdots \\
D_{n-1}^{*}
\end{array}\right]
\end{aligned}
$$


and hence

$$
\Gamma \Gamma^{*}=\left[\begin{array}{lll}
T_{1} \Gamma_{1} & \cdots & T_{n-1} \Gamma_{n-1}
\end{array}\right] D^{*} D\left[\begin{array}{c}
\Gamma_{1}^{*} T_{1}^{*} \\
\vdots \\
\Gamma_{n-1}^{*} T_{n-1}^{*}
\end{array}\right] \leq \sum_{j=1}^{n-1} T_{j} \Gamma_{j} \Gamma_{j}^{*} T_{j}^{*},
$$

as $D$ is a contraction. This implies

$$
\sum_{j=1}^{n-1}\left(I_{\mathcal{B}(\mathcal{H})}-C_{T_{j}}\right)\left(\Gamma_{j} \Gamma_{j}^{*}\right)=\sum_{j=1}^{n-1} \Gamma_{j} \Gamma_{j}^{*}-\sum_{j=1}^{n-1} T_{j} \Gamma_{j} \Gamma_{j}^{*} T_{j}^{*} \leq 0 .
$$

Next, for each natural number $m$ and $j=1, \ldots, n-1$, we have

$$
\begin{aligned}
\Sigma_{\hat{T}_{j, n}}^{m}\left(\Gamma_{j} \Gamma_{j}^{*}\right)-T_{j}^{m} \Sigma_{\hat{T}_{j, n}}\left(\Gamma_{j} \Gamma_{j}^{*}\right) T_{j}^{* m} & \leq \Sigma_{\hat{T}_{j, n}}^{m}\left(\Gamma_{j} \Gamma_{j}^{*}\right)-T_{j}^{m} \Sigma_{\hat{T}_{j, n}}^{m}\left(\Gamma_{j} \Gamma_{j}^{*}\right) T_{j}^{* m} \\
& =\Sigma_{\hat{T}_{j, n}^{m}}^{m}\left(I_{\mathcal{B}(\mathcal{H})}-C_{T_{j}^{m}}\right)\left(\Gamma_{j} \Gamma_{j}^{*}\right),
\end{aligned}
$$

and since (see (3.11)

$$
\sum_{\hat{T}_{j, n}}^{m}\left(I_{\mathcal{B}(\mathcal{H})}-C_{T_{j}^{m}}\right)=\Sigma_{\hat{T}_{j, n}}^{m} \Sigma_{T_{j}}^{m}\left(I_{\mathcal{B}(\mathcal{H})}-C_{T_{j}}\right)=\Sigma_{\hat{T}_{n}}^{m}\left(I_{\mathcal{B}(\mathcal{H})}-C_{T_{j}}\right),
$$

it follows that

$$
\sum_{j=1}^{n-1}\left[\Sigma_{\hat{T}_{j, n}}^{m}\left(\Gamma_{j} \Gamma_{j}^{*}\right)-T_{j}^{m} \Sigma_{\hat{T}_{j, n}}\left(\Gamma_{j} \Gamma_{j}^{*}\right) T_{j}^{* m}\right] \leq \Sigma_{\hat{T}_{n}}^{m}\left(\sum_{j=1}^{n-1}\left(I_{\mathcal{B}(\mathcal{H})}-C_{T_{j}}\right)\left(\Gamma_{j} \Gamma_{j}^{*}\right)\right) \leq 0 .
$$

Since $T_{j}$ is pure, passing to the limit as $m \rightarrow \infty$, we get

$$
\sum_{j=1}^{n-1} \Sigma_{\hat{T}_{j, n}}\left(\Gamma_{j} \Gamma_{j}^{*}\right) \leq 0
$$

On the other hand, since $\Sigma_{\hat{T}_{j, n}}\left(\Gamma_{j} \Gamma_{j}^{*}\right) \geqslant 0$, by definition, we have that

$$
\Sigma_{\hat{T}_{j, n}}\left(\Gamma_{j} \Gamma_{j}^{*}\right)=0,
$$

for all $j=1, \ldots, n-1$. Hence

$$
\left.F_{j}^{*}\right|_{\mathcal{F}_{j}}-\Pi^{*} \Psi_{j}=\Gamma_{j}=0 \text { and } \tilde{F}_{n-1}^{*}-\Pi^{*} \Psi_{n-1}=\Gamma_{n-1}=0,
$$

$j=1, \ldots, n-2$. Finally, for $\boldsymbol{p} \in \mathbb{Z}_{+}^{n-1}$ and $\eta \in \mathcal{D}_{\hat{T}_{n}}$, we have

$$
\Pi^{*} M_{\Phi}\left(\boldsymbol{z}^{p} \otimes \eta\right)=\Pi^{*} M_{\boldsymbol{z}^{p}} M_{\Phi}(1 \otimes \eta)=\hat{T}_{n}^{p} \Pi^{*} M_{\Phi}(1 \otimes \eta)=\hat{T}_{n}^{p} \Pi^{*} M_{\Phi} Q \eta .
$$

But

$$
\hat{T}_{n}^{p} \Pi^{*} M_{\Phi} Q \eta=\hat{T}_{n}^{p} \Pi^{*}\left[Q A^{*}+\sum_{j=1}^{n-1} M_{z_{j}} \Psi_{j} B_{j}^{*}\right] \eta=\hat{T}_{n}^{p}\left[D_{\hat{T}_{n}} A^{*}+\sum_{j=1}^{n-1} T_{j} \Pi^{*} \Psi_{j} B_{j}^{*}\right] \eta,
$$

by Lemma 3.2 and (4.1), and therefore by (3.8) and Lemma 4.2

$$
\Pi^{*} M_{\Phi}\left(\boldsymbol{z}^{p} \otimes \eta\right)=\hat{T}_{n}^{p} T_{n} D_{\hat{T}_{n}} \eta=T_{n} \hat{T}_{n}^{p} D_{\hat{T}_{n}} \eta=T_{n} \Pi^{*}\left(\boldsymbol{z}^{p} \otimes \eta\right) .
$$


But since $\left\{\boldsymbol{z}^{\boldsymbol{p}} \otimes \eta: \boldsymbol{p} \in \mathbb{Z}_{+}^{n-1}, m \in \mathcal{D}_{\hat{T}_{n}}\right\}$ is a total set in $H_{\mathcal{D}_{\hat{T}_{n}}}^{2}\left(\mathbb{D}^{n-1}\right)$, we conclude

$$
\Pi^{*} M_{\Phi}=T_{n} \Pi^{*}
$$

which completes the proof of the theorem.

We are now ready to prove the dilation theorem for commuting tuples in $\mathcal{P}_{n}(\mathcal{H}$ ) (recall Definitions 1.1 and 1.2). Recall from Theorem 4.1 that given a $T \in \mathcal{P}_{n}(\mathcal{H})$, the canonical isometric dilation $\Pi: \mathcal{H} \rightarrow H_{\mathcal{D}_{\hat{T}_{n}}}^{2}\left(\mathbb{D}^{n-1}\right)$ of the $(n-1)$-tuple $\hat{T}_{n}$ is an isometry and

$$
\Pi T_{i}^{*}=M_{z_{i}}^{*} \Pi
$$

for all $i=1, \ldots, n-1$.

Theorem 4.4. If $T \in \mathcal{P}_{n}(\mathcal{H})$, then there exists a contractive multiplier $\Phi \in \mathcal{S}_{n-1}\left(\mathcal{D}_{\hat{T}_{n}}, \mathcal{D}_{\hat{T}_{n}}\right)$ such that

$$
\Pi T_{i}^{*}= \begin{cases}M_{z_{i}}^{*} \Pi & \text { if } i=1, \ldots, n-1, \\ M_{\Phi}^{*} \Pi & \text { if } i=n .\end{cases}
$$

If, in addition, $T$ is finite rank, then $\Phi$ is an inner function. In particular, a finite rank $T$ in $\mathcal{P}_{n}(\mathcal{H})$ dilates to a commuting isometries $\left(M_{z_{1}}, \ldots, M_{z_{n-1}}, M_{\Phi}\right)$ on $H_{\mathcal{D}_{\hat{T}_{n}}}^{2}\left(\mathbb{D}^{n-1}\right)$.

Proof. Let $\left\{G_{1}, \ldots, G_{n-1}\right\}$ be the positive operators on $\mathcal{H}$ corresponding to $T \in \mathcal{P}_{n}(\mathcal{H})$. Then

$$
I-T_{n} T_{n}^{*}=G_{1}+\cdots+G_{n-1},
$$

so that

$$
\begin{aligned}
D_{\hat{T}_{n}}^{2}-T_{n} D_{\hat{T}_{n}}^{2} T_{n}^{*} & =\sum_{\boldsymbol{k} \in \mathbb{Z}_{+}^{n-1}}(-1)^{|\boldsymbol{k}|} \hat{T}_{n}^{\boldsymbol{k}}\left(I-T_{n} T_{n}^{*}\right) \hat{T}_{n}^{* \boldsymbol{k}} \\
& =\prod_{j=1}^{n-1}\left(I_{\mathcal{B}(\mathcal{H})}-C_{T_{j}}\right)\left(I-T_{n} T_{n}^{*}\right) \\
& =\sum_{i=1}^{n-1} \prod_{j=1}^{n-1}\left(I_{\mathcal{B}(\mathcal{H})}-C_{T_{j}}\right)\left(G_{i}\right) \\
& =\sum_{i=1}^{n-1} \prod_{\substack{j=1 \\
j \neq i}}^{n-1}\left(I_{\mathcal{B}(\mathcal{H})}-C_{T_{j}}\right)\left(I_{\mathcal{B}(\mathcal{H})}-C_{T_{i}}\right)\left(G_{i}\right) \\
& =\sum_{i=1}^{n-1}\left(\prod_{\substack{j=1 \\
j \neq i}}^{n-1}\left(I_{\mathcal{B}(\mathcal{H})}-C_{T_{j}}\right)\left(G_{i}\right)-T_{i}\left(\prod_{\substack{j=1 \\
j \neq i}}^{n-1}\left(I_{\mathcal{B}(\mathcal{H})}-C_{T_{j}}\right)\left(G_{i}\right)\right) T_{i}^{*}\right)
\end{aligned}
$$

in view of $C_{T_{p}} C_{T_{q}}=C_{T_{q}} C_{T_{p}}$ for all $p, q=1, \ldots, n-1$. If we define (see Definition 1.1)

$$
S_{T}\left(G_{i}\right)=\prod_{\substack{j=1 \\ j \neq i}}^{n-1}\left(I_{\mathcal{B}(\mathcal{H})}-C_{T_{j}}\right)\left(G_{i}\right)
$$


then $S_{T}\left(G_{i}\right) \geq 0$ for all $i=1, \ldots, n-1$, by assumption, and

$$
D_{\hat{T}_{n}}^{2}-T_{n} D_{\hat{T}_{n}}^{2} T_{n}^{*}=\sum_{i=1}^{n-1}\left(S_{T}\left(G_{i}\right)-T_{i} S_{T}\left(G_{i}\right) T_{i}^{*}\right) .
$$

If we let $F_{i}^{2}=S_{T}\left(G_{i}\right)$ for all $i=1, \ldots, n-1$, for simplicity, then

$$
D_{\hat{T}_{n}}^{2}+\sum_{i=1}^{n-1} T_{i} F_{i}^{2} T_{i}^{*}=T_{n} D_{\hat{T}_{n}}^{2} T_{n}^{*}+\sum_{i=1}^{n-1} F_{i}^{2},
$$

and so the map

$$
U:\left\{\left(D_{\hat{T}_{n}} h, F_{1} T_{1}^{*} h, \ldots, F_{n-1} T_{n-1}^{*} h\right): h \in \mathcal{H}\right\} \rightarrow\left\{\left(D_{\hat{T}_{n}} T_{n}^{*} h, F_{1} h, \ldots, F_{n-1} h\right): h \in \mathcal{H}\right\}
$$

defined by

$$
U\left(D_{\hat{T}_{n}} h, F_{1} T_{1}^{*} h, \ldots, F_{n-1} T_{n-1}^{*} h\right)=\left(D_{\hat{T}_{n}} T_{n}^{*} h, F_{1} h, \ldots, F_{n-1} h\right),
$$

for all $h \in \mathcal{H}$, is an isometry. Set

$$
\mathcal{F}_{i}=\overline{\operatorname{Ran}} F_{i}
$$

for all $i=1, \ldots, n-1$. Then, by adding an infinite dimensional Hilbert space $\mathcal{K}$, if necessary, one can find a unitary

$$
U: \mathcal{D}_{\hat{T}_{n}} \oplus\left(\oplus_{i=1}^{n-1} \mathcal{F}_{i}\right) \oplus \mathcal{K} \rightarrow \mathcal{D}_{\hat{T}_{n}} \oplus\left(\oplus_{i=1}^{n-1} \mathcal{F}_{i}\right) \oplus \mathcal{K}
$$

such that

$$
U\left(D_{\hat{T}_{n}} h, F_{1} T_{1}^{*} h, \ldots, F_{n-1} T_{n-1}^{*} h, 0_{\mathcal{K}}\right)=\left(D_{\hat{T}_{n}} T_{n}^{*} h, F_{1} h, \ldots, F_{n-1} h, 0_{\mathcal{K}}\right),
$$

for all $h \in \mathcal{H}$. Finally, since

$$
\Sigma_{\hat{T}_{i, n}}^{N}\left(F_{i} F_{i}^{*}\right)=\prod_{k \neq i, n}\left(I_{\mathcal{B}(\mathcal{H})}-C_{T_{k}^{N}}\right)\left(G_{i}\right) \leq G_{i}
$$

implies that $\Sigma_{\hat{T}_{i, n}}\left(F_{i} F_{i}^{*}\right)$ exists for all $i=1, \ldots, n-1$, the first part of the theorem follows from Theorem 4.3 ,

In addition now assume that $T$ is finite rank. Then $\mathcal{D}_{\hat{T}_{n}}$ and $\mathcal{F}_{i}, i=1, \ldots, n-1$, are all finite dimensional Hilbert spaces. Then the unitary $U$ in (4.2) extends to a unitary on $\mathcal{D}_{\hat{T}_{n}} \oplus\left(\oplus_{i=1}^{n-1} \mathcal{F}_{i}\right)$ which we also denote by $U$ and then by applying Theorem 4.3 (with $\mathcal{K}=0$ ), we have

$$
M_{\Phi}^{*} \Pi=\Pi T_{n}^{*},
$$

where $\Phi$ is the transfer function corresponding to $U^{*}$. In this case, Lemma 3.1 yields that $\Phi$ is a $\mathcal{B}\left(D_{\hat{T}_{n}}\right)$-valued inner multiplier. This completes the proof of the theorem.

Remark 4.5. It is worth mentioning that the converse of the above theorem is also true. Indeed, if $\mathcal{E}$ is a Hilbert space, $\Phi \in \mathcal{S}_{n-1}(\mathcal{E}, \mathcal{E})$ and $\mathcal{Q} \subseteq H_{\mathcal{E}}^{2}\left(\mathbb{D}^{n-1}\right)$ is a joint invariant subspace for $\left(M_{z_{1}}^{*}, \ldots, M_{z_{n-1}}^{*}, M_{\Phi}^{*}\right)$, then [4, Theorem 5.1] implies that

$$
\left(\left.P_{\mathcal{Q}} M_{z_{1}}\right|_{\mathcal{Q}}, \ldots,\left.P_{\mathcal{Q}} M_{z_{n-1}}\right|_{\mathcal{Q}},\left.P_{\mathcal{Q}} M_{\Phi}\right|_{\mathcal{Q}}\right) \in \mathcal{P}_{n}(\mathcal{Q})
$$




\section{VON-NEUMANN INEQUALITY FOR FINITE RANK TUPLES IN $\mathcal{P}_{n}(\mathcal{H})$}

Now we turn to the von-Neumann inequality for finite rank tuples in $\mathcal{P}_{n}(\mathcal{H})$.

Theorem 5.1. Let $T \in \mathcal{P}_{n}(\mathcal{H})$ be a finite rank tuple. Then there exists an algebraic variety $V$ in $\overline{\mathbb{D}}^{n}$ such that

$$
\|p(T)\| \leq \sup _{\boldsymbol{z} \in V}|p(\boldsymbol{z})|
$$

for all $p \in \mathbb{C}\left[z_{1}, \ldots, z_{n}\right]$. If, in addition, $T_{n}$ is a pure contraction, then $V \subseteq \mathbb{D}^{n}$.

Proof. Let $\left(M_{z_{1}}, \ldots, M_{z_{n-1}}, M_{\Phi\left(z_{1}, \ldots, z_{n-1}\right)}\right)$ on $H_{D_{\hat{T}_{n}}}^{2}\left(\mathbb{D}^{n-1}\right)$ be the isometric dilation of $T$ as in Theorem 4.4 and let

$$
\Phi(\boldsymbol{z})=A^{*}+C^{*} E(\boldsymbol{z})\left(I_{\mathcal{F}}-D^{*} E(\boldsymbol{z})\right)^{-1} B^{*} \quad\left(\boldsymbol{z} \in \mathbb{D}^{n-1}\right),
$$

the transfer function corresponding to the unitary

$$
U^{*}=\left[\begin{array}{ll}
A^{*} & C^{*} \\
B^{*} & D^{*}
\end{array}\right]: D_{\hat{T}_{n}} \oplus \mathcal{F} \rightarrow D_{\hat{T}_{n}} \oplus \mathcal{F}
$$

as constructed in the proof of Theorem 4.4 (see (4.3) and note, in view of the assumption that $T$ is finite rank, that $\mathcal{K}=\{0\})$. Let

$$
A^{*}=\left[\begin{array}{cc}
W^{*} & 0 \\
0 & E^{*}
\end{array}\right] \text { on } \mathcal{H}_{0} \oplus \mathcal{H}_{1}=D_{\hat{T}_{n}},
$$

be the canonical decomposition of $A^{*}$ into the unitary part $W^{*}$ on $\mathcal{H}_{0}$ and the completely non-unitary part $E^{*}$ on $\mathcal{H}_{1}$. With respect to the above decomposition of $A^{*}$, let

$$
\Phi(\boldsymbol{z})=\left[\begin{array}{cc}
\Phi_{0}(\boldsymbol{z}) & 0 \\
0 & \Phi_{1}(\boldsymbol{z})
\end{array}\right]
$$

be the decomposition of $\Phi$, where

$$
\Phi_{0}(\boldsymbol{z}) \equiv W^{*} \quad\left(\boldsymbol{z} \in \mathbb{D}^{n-1}\right),
$$

and

$$
\Phi_{1}(\boldsymbol{z})=E^{*}+C^{*} E(\boldsymbol{z})\left(I_{\mathcal{F}}-D^{*} E(\boldsymbol{z})\right)^{-1} B^{*} \quad\left(\boldsymbol{z} \in \mathbb{D}^{n-1}\right),
$$

is a multiplier in $H_{\mathcal{B}\left(\mathcal{H}_{1}\right)}^{\infty}\left(\mathbb{D}^{n-1}\right)$. We set

$$
V_{0}:=\left\{\boldsymbol{z} \in \overline{\mathbb{D}}^{n}: \operatorname{det}\left(z_{n} I_{\mathcal{H}_{0}}-\Phi_{0}\left(z_{1}, \ldots, z_{n-1}\right)\right)=0\right\}=\mathbb{D}^{n-1} \times \sigma\left(W^{*}\right),
$$

and

$$
V_{1}:=\left\{\boldsymbol{z} \in \mathbb{D}^{n}: \operatorname{det}\left(z_{n} I_{\mathcal{H}_{1}}-\Phi_{1}\left(z_{1}, \ldots, z_{n-1}\right)\right)=0\right\} .
$$

Then for each $p \in \mathbb{C}\left[z_{1}, \ldots, z_{n}\right]$ we have

$$
\begin{aligned}
\|p(T)\| & \leq\left\|p\left(M_{z_{1}}, \ldots, M_{z_{n-1}}, M_{\Phi\left(z_{1}, \ldots, z_{n-1}\right)}\right)\right\| \\
& =\left\|M_{p\left(z_{1}, \ldots, z_{n-1}, \Phi\left(z_{1}, \ldots, z_{n-1}\right)\right)}\right\| \\
& =\sup _{\theta_{1}, \ldots, \theta_{n-1}}\left\|p\left(e^{i \theta_{1}} I_{D_{\hat{T}_{n}}}, \ldots, e^{i \theta_{n-1}} I_{D_{\hat{T}_{n}}}, \Phi\left(e^{i \theta_{1}}, \ldots, e^{i \theta_{n-1}}\right)\right)\right\| .
\end{aligned}
$$


Moreover, for $j=0,1$, we have

$$
\begin{aligned}
& \sup _{\theta_{1}, \ldots, \theta_{n-1}}\left\|p\left(e^{i \theta_{1}} I_{H_{j}}, \ldots, e^{i \theta_{n-1}} I_{H_{j}}, \Phi_{j}\left(e^{i \theta_{1}}, \ldots, e^{i \theta_{n-1}}\right)\right)\right\| \\
& =\sup _{\theta_{1}, \ldots, \theta_{n-1}}\left\{\left|p\left(e^{i \theta_{1}}, \ldots, e^{i \theta_{n-1}}, \lambda\right)\right|: \lambda \in \sigma\left(\Phi_{j}\left(e^{i \theta_{1}}, \ldots, e^{i \theta_{n-1}}\right)\right)\right\} \\
& \leq\|p\|_{\partial V_{j}} .
\end{aligned}
$$

Since $\Phi\left(e^{i \theta_{1}}, \ldots, e^{i \theta_{n-1}}\right)=\Phi_{0}\left(e^{i \theta_{1}}, \ldots, e^{i \theta_{n-1}}\right) \oplus \Phi_{1}\left(e^{i \theta_{1}}, \ldots, e^{i \theta_{n-1}}\right)$, we have by continuity and Lemma 3.1 that

$$
\|p(T)\| \leq \sup _{\boldsymbol{z} \in V}|p(\boldsymbol{z})|
$$

where

$$
V=V_{0} \cup V_{1}
$$

For the second part, we prove that $\mathcal{H}_{0}=\{0\}$ which would imply that $V_{0}$ is the empty set. Let $\mathcal{Q}=\Pi(\mathcal{H})$, where $\Pi$ is the canonical dilation map for $\hat{T}_{n}$. We claim that

$$
\mathcal{Q} \subseteq H_{\mathcal{H}_{1}}^{2}\left(\mathbb{D}^{n-1}\right) .
$$

Indeed, let $g \in H_{\mathcal{H}_{0}}^{2}\left(\mathbb{D}^{n-1}\right), m \in \mathbb{Z}_{+}$and let $g_{m}=M_{\Phi_{0}}^{* m} g$. Then $g_{m} \in H_{\mathcal{H}_{0}}^{2}\left(\mathbb{D}^{n-1}\right)$ and

$$
g=M_{\Phi_{0}}^{m} g_{m}
$$

For $f \in \mathcal{Q}$, we have

$$
\langle g, f\rangle=\left\langle M_{\Phi_{0}}^{m} g_{m}, f\right\rangle=\left\langle g_{m}, M_{\Phi_{0}}^{* m} f\right\rangle=\left\langle g_{m}, M_{\Phi}^{* m} f\right\rangle=\left\langle g_{m}, T_{n}^{* m} f\right\rangle \rightarrow 0,
$$

as $m \rightarrow \infty$, as $T_{n}$ is pure. This implies that $\mathcal{Q} \subseteq H_{\mathcal{H}_{1}}^{2}\left(\mathbb{D}^{n-1}\right)$. By the minimality of the isometric dilation of $\hat{T}_{n}$ (see Theorem 4.1), we have

$$
\bigvee_{k \in \mathbb{Z}_{+}^{n-1}} M_{z}^{k} \mathcal{Q}=H_{\mathcal{D}_{\hat{T}_{n}}}^{2}\left(\mathbb{D}^{n-1}\right) .
$$

Clearly, $H_{\mathcal{H}_{1}}^{2}\left(\mathbb{D}^{n-1}\right) \subseteq H_{\mathcal{D}_{\hat{T}_{n}}}^{2}\left(\mathbb{D}^{n-1}\right)$ is a joint reducing subspace for $\left(M_{z_{1}}, \ldots, M_{z_{n-1}}\right)$. Then we have $H_{\mathcal{H}_{0}}^{2}\left(\mathbb{D}^{n-1}\right)=\{0\}$ and hence, $\mathcal{H}_{0}=\{0\}$. This completes the proof of the theorem.

The finiteness assumption for tuples in $\mathcal{P}_{n}(\mathcal{H})$ seems natural for refined (in terms of algebraic varieties) von Neumann inequality. However, we do not know how to prove the existence of (explicit) isometric dilations for tuples without the finiteness assumption.

Acknowledgement: The research of the second named author is supported by DST-INSPIRE Faculty Fellowship No. DST/INSPIRE/04/2015/001094. The research of the third named author is supported in part by NBHM grant NBHM/R.P.64/2014, and the Mathematical Research Impact Centric Support (MATRICS) grant, File No: MTR/2017/000522 and Core Research Grant, File No: CRG/2019/000908, by the Science and Engineering Research Board (SERB), Department of Science \& Technology (DST), Government of India. 


\section{REFERENCES}

[1] J. Agler and J. McCarthy, Distinguished Varieties, Acta. Math. 194 (2005), 133-153.

[2] C. G. Ambrozie, M. Engliš ans V. Müller, Operator tuples and analytic models over general domains in $\mathbb{C}^{n}$, J. Operator Theory 47 (2002), 287-302.

[3] T. Ando, On a pair of commutative contractions, Acta Sci. Math. (Szeged) 24 (1963), 88-90.

[4] J. A. Ball, W.S. Li, D. Timotin and T. T. Trent, A commutant lifting theorem on the polydisc, Indiana Univ. Math. J. 48 (1999), 653-675.

[5] S. Barik, B.K. Das, K.J. Haria and J. Sarkar, Isometric dilations and von Neumann inequality for a class of tuples in the polydisc, Trans. Amer. Math. Soc. 372 (2019), 1429-1450.

[6] M.-D. Choi and K. R. Davidson, A $3 \times 3$ dilation counterexample, Bull. London Math. Soc. 45 (2013), $511-519$.

[7] M. Crabb and A. Davie, Von Neumann's inequality for Hilbert space operators, Bull. London Math. Soc. 7 (1975), 49-50.

[8] R. E. Curto and F.-H. Vasilescu, Standard operator models in the polydisc, Indiana Univ. Math. J. 42 (1993), 791-810.

[9] R. E. Curto and F.-H. Vasilescu, Standard operator models in the polydisc, II, Indiana Univ. Math. J. 44 (1995), 727-746.

[10] B. K. Das and J. Sarkar, Ando dilations, von Neumann inequality, and distinguished varieties, J. Funct. Anal. 272 (2017), 2114-2131.

[11] B. K. Das, J. Sarkar and S. Sarkar, Factorizations of contractions, Adv. Math 322 (2017), 186-200.

[12] S. Drury, Remarks on von Neumann's inequality, Banach spaces, harmonic analysis, and probability theory, Storrs, CT, 1980/1981, Lecture Notes in Mathematics 995 (Springer, Berlin, 1983) 14-32.

[13] C. Foias and A. Frazho, The commutant lifting approach to interpolation problems, Operator Theory: Advances and Applications, 44. Birkhuser Verlag, Basel, 1990.

[14] A. Grinshpan, D.S. Kaliuzhnyi-Verbovetskyi, V. Vinnikov and H.J. Woerdeman, Classes of tuples of commuting contractions satisfying the multivariable von Neumann inequality, J. Funct. Anal. 256 (2009), 3035-3054.

[15] J. A. Holbrook, Inequalities of von Neumann type for small matrices, Function spaces, Edwardsville, IL, 1990, Lecture Notes in Pure and Applied Mathematics 136 (Dekker, New York, 1992), 189-193.

[16] J. A. Holbrook, Schur norms and the multivariate von Neumann inequality, Recent advances in operator theory and related topics, Szeged, 1999, Operator Theory: Advances and Applications 127 (Birkhäuser, Basel, 2001), 375-386.

[17] G. Knese, The von Neumann inequality for $3 \times 3$ matrices, Bull. Lond. Math. Soc. 48 (2016), 53-57.

[18] E. Kosiński, Three-point Nevanlinna Pick problem in the polydisc, Proc. Lond. Math. Soc. 111 (2015), 887-910.

[19] V. Müller and F.-H. Vasilescu, Standard models for some commuting multioperators, Proc. Amer. Math. Soc. 117 (1993), 979-989.

[20] D. Sarason, Generalized interpolation in $H^{\infty}$, Trans. Amer. Math. Soc. 127 (1967), 179-203.

[21] B. Sz.-Nagy and C. Foias, Harmonic Analysis of Operators on Hilbert Space. North-Holland, AmsterdamLondon, 1970.

[22] S. Parrott, Unitary dilations for commuting contractions, Pacific J. Math. 34 (1970), 481-490.

[23] N. Th. Varopoulos, On an inequality of von Neumann and an application of the metric theory of tensor products to operator theory, J. Funct. Anal. 16 (1974), 83-100. 
Department of Mathematics, Indian Institute of Technology Bombay, Powai, Mumbai, 400076, INDIA

E-mail address: sibaprasadbarik00@gmail.com

Department of Mathematics, Indian Institute of Technology Bombay, Powai, Mumbai, 400076, INDIA

E-mail address: dasb@math.iitb.ac.in, bata436@gmail.com

Indian Statistical Institute, Statistics and Mathematics Unit, 8th Mile, Mysore Road, BANGALORE, 560059, INDIA

E-mail address: jay@isibang.ac.in, jaydeb@gmail.com 